\title{
Disordered expression of HOX genes in human non-small cell lung cancer
}

\author{
MOTOKI ABE ${ }^{1,2}$, JUN-ICHI HAMADA ${ }^{1}$, OSAMU TAKAHASHI ${ }^{1,2}$, YOKO TAKAHASHI ${ }^{1}$, MITSUHIRO TADA ${ }^{1}$, \\ MASAKI MIYAMOTO ${ }^{2}$, TOSHIAKI MORIKAWA ${ }^{2}$, SATOSHI KONDO $^{2}$ and TETSUYA MORIUCHI ${ }^{1}$ \\ ${ }^{1}$ Division of Cancer-Related Genes, Institute for Genetic Medicine, Hokkaido University; ${ }^{2}$ Department of Surgical Oncology, \\ Division of Cancer Medicine, Hokkaido University Graduate School of Medicine, Sapporo 060-0815, Japan
}

Received October 11, 2005; Accepted December 8, 2005

\begin{abstract}
We hypothesized that the disordered tissue architecture in cancer results from the cells executing the program designed during ontogeny in a spatio-temporally inappropriate manner. HOX genes are known as master regulators of embryonic morphogenesis, and encode transcription factors which regulate the transcription of the downstream genes to realize the program of body plan. In this study, we quantified the expression levels of 39 HOX genes in 41 human non-small cell lung cancer (non-SCLC) and noncancerous lung tissues by a comprehensive analysis system based on the real-time RT-PCR method. We found that the expression levels of $H O X A 1, A 5, A 10$ and $C 6$ in squamous cell carcinoma tissues (and HOXA5 and A10 in adenocarcinoma tissues) were significantly higher than those in the non-cancerous tissues. Comparison of HOX gene expressions between adenocarcinoma and squamous cell carcinoma tissues showed higher expressions of HOXA1, D9, D10 and D11 in squamous cell carcinoma tissues than in adenocarcinoma tissues. Immunohistochemical analysis revealed that HOXA5 and A10 proteins were localized in the cytoplasm of tumor cells in both adenocarcinoma and squamous cell carcinoma tissues. These results suggest that the disordered patterns of HOX gene expressions were involved not only in the development of non-SCLC but also in the histologically aberrant diversity such as adenocarcinoma and squamous cell carcinoma.
\end{abstract}

\section{Introduction}

It can be hypothesized that the disordered tissue architecture in cancer results from the cells executing the program designed during ontogeny in a spatio-temporally inappropriate

Correspondence to: Dr Jun-ichi Hamada, Division of CancerRelated Genes, Institute for Genetic Medicine, Hokkaido University, Kita-15, Nishi-7, Kita-ku, Sapporo 060-0815, Japan

E-mail: jhamada@igm.hokudai.ac.jp

Key words: homeobox gene, HOX, real-time RT-PCR, lung cancer manner. In other words, the cancer cells may partly follow the ontogenic program to display the transformed phenotype and dynamic movement to surrounding or distant areas. Homeobox genes are a superfamily of the genes encoding transcription factors, which regulate the embryonic morphogenesis in animals (1). The clustered group of homeobox genes in human are called HOX genes. The genes contain a 180-bp DNA sequence (homeobox), which encodes a highly conserved 60 amino-acid homeodomain. The HOX proteins function as transcription factors through their homeodomain, which is responsible for recognition and binding of sequencespecific DNA motifs $(2,3)$. In human genome, 39 HOX genes are clustered in a similar arrangement of 13 paralog groups in four different chromosomal regions, HOXA, B, C, and D (4). During embryonic morphogenesis, HOX gene expressions are regulated in a precise spatiotemporal manner: the 3'-end genes of the clusters are expressed earlier and in more anterior domain than the genes localized in more 5' positions (5).

There is a growing interest in the relationship of inappropriate expressions of HOX genes with carcinogenesis or malignant progression. Some HOX genes exhibit different expression levels in a variety of human cancers including kidney, colon, bladder, melanoma and prostate cancers, compared to normal tissues from which they were derived (6-10). For example, HOXB5 and B9 are expressed in normal kidney but not in renal cancer, whereas the expression of HOXC11 is observed in human renal cancer but not in normal kidney (6). In human prostate cancer, overexpression of $\mathrm{HOXC} 8$ correlates with loss of differentiation phenotype (10). Increased expressions of HOXC4, C5, C6 and C11 are likely to be involved in the development of human bladder transitional cell carcinomas (8). We also found that the expression levels of $H O X A 11, A 13, B 9, D 12$ and D13 were higher in cutaneous melanoma than in nevus pigmentosus (9).

There are few studies on HOX gene expressions in human lung cancer. The expression of some HOX genes in human small-cell lung cancers xenografted into nude mice was different from that in normal lung (11). Analysis of HOX gene expressions by using degenerate reverse transcriptionPCR showed that HOXA9, A10 and B9 were frequently upregulated in human lung cancer cell lines and lung tumor tissues (12). Lechner et al reported that some HOX genes of which expression was restricted to pulmonary embryogenesis were re-expressed in lung cancer cells (13). To better 
understand the roles of HOX genes in lung cancer, we investigated the HOX gene expressions in 41 human non-small cell lung cancer tissues by a comprehensive analysis system to quantify the expressions of 39 human $H O X$ genes based on the real-time reverse transcription-PCR method. We also examined the relationship between HOX gene expression and clinicopathologic parameters.

\section{Materials and methods}

Patients and samples. A total of 41 primary lung cancer tissues (28 adenocarcinoma and 13 squamous cell carcinoma tissues) and 15 non-cancerous tissues were obtained from surgical specimens resected from patients who underwent radical surgery without any preoperative chemotherapy or radiotherapy at the Department of Surgical Oncology, Hokkaido University Hospital and 33 affiliated hospitals in Hokkaido, Japan, between January 2001 and September 2004. Only those patients who agreed with the aim and contents of this study and who provided their written informed consent were included in the study. Tumor and normal tissue samples of $\sim 5 \mathrm{~mm}$-size were immediately cut from the lung resected by a standard surgical procedure, snap-frozen in liquid nitrogen, and stored at $-80^{\circ} \mathrm{C}$ until use. Part of each tumor sample piece was cut and examined for histopathological analysis, and the sample pieces were used for RNA extraction. All procedures in this portion of the study were approved by the Ethics Committee of Hokkaido University and the independent internal ethics committees of the affiliated hospitals.

Histological subclassification and staging of the tumors were done by reviewing the specimens taken for pathological diagnosis, according to the TNM classification. The tumor status of each case was categorized based on the pTNM pathological classification of the International Union Against Cancer (14). Pertinent major clinicopathological parameters are shown in Table I.

RNA extraction and cDNA preparation. Total RNA was extracted from powdery frozen tissue of each clinical specimen which had been crushed in liquid nitrogen with a CRYO-Press compressor (Microtec Nition, Chiba, Japan) with Trizol reagent (Invitrogen, Carlsbad, CA) according to the manufacturer's instructions. For exclusion of contaminated genomic DNA, $50 \mu \mathrm{g}$ of total RNA was incubated for $30 \mathrm{~min}$ at $37^{\circ} \mathrm{C}$ in $50 \mu \mathrm{l}$ of reaction mixture containing $40 \mathrm{mM}$ Tris$\mathrm{HCl}$ (pH 7.2), $10 \mathrm{mM} \mathrm{NaCl}, 6 \mathrm{mM} \mathrm{MgCl}, 2 \mathrm{mM}$ dithiothreitol, $0.04 \mathrm{U} / \mu 1 \mathrm{PQ} 1 \mathrm{DNase}$ (Promega, Madison, WI), and $0.4 \mathrm{U} / \mu 1$ RNase inhibitor. Reverse transcription reaction for real-time PCR was performed by the same method as described in our previous study (15).

Quantitative real-time PCR. Quantitative RT-PCR assays were carried out by using ABI PRISM-7900HT (Applied Biosystems) with SYBR-green fluorescence under the same condition as described previously (15). The primer sets for amplification of 39 HOX genes and $B$-actin gene were listed in our previous study (15).

Immunohistochemistry. Surgical specimens were fixed in a $10 \%$ formalin solution and embedded by routine methods in
Table I. Clinicopathologic parameters.

\begin{tabular}{|c|c|c|}
\hline Parameters & $\begin{array}{l}\text { Adenocarcinoma } \\
(\mathrm{n}=28)\end{array}$ & $\begin{array}{c}\text { Squamous cell } \\
\text { carcinoma }(\mathrm{n}=13)\end{array}$ \\
\hline Age, median (range) & $68(39-82)$ & $72(47-79)$ \\
\hline \multicolumn{3}{|l|}{ Gender } \\
\hline Male & 16 & 12 \\
\hline Female & 12 & 1 \\
\hline \multicolumn{3}{|l|}{ Differentiation } \\
\hline Well & 8 & 1 \\
\hline Moderately & 11 & 10 \\
\hline Poorly & 7 & 2 \\
\hline \multicolumn{3}{|l|}{ pTNM } \\
\hline pT2N0M0 & 20 & 7 \\
\hline pT2N1M0 & 5 & 3 \\
\hline pT2N2M0 & 3 & 3 \\
\hline \multicolumn{3}{|l|}{ pStage } \\
\hline IB & 20 & 7 \\
\hline IIB & 5 & 3 \\
\hline IIIA & 3 & 3 \\
\hline \multicolumn{3}{|l|}{ Smoking } \\
\hline+ & 14 & 10 \\
\hline- & 12 & 2 \\
\hline \multicolumn{3}{|l|}{ Brinkman's index } \\
\hline$\geq 600$ & 5 & 6 \\
\hline$<600$ & 21 & 6 \\
\hline \multicolumn{3}{|l|}{$\begin{array}{l}\text { Tumor marker, } \\
\text { median (range) }\end{array}$} \\
\hline CEA (ng/ml) & $5.1(0.6-48.0)$ & $3.2(1.7-16.6)$ \\
\hline $\mathrm{SCC}(\mathrm{ng} / \mathrm{ml})$ & $0.5(0-1.5)$ & $1.4(0.7-2.3)$ \\
\hline NSE (ng/ml) & $4.6(0-9.7)$ & $8.2(0-11.9)$ \\
\hline CYFRA21-1 (ng/ml) & $0.9(0-7.0)$ & $5.2(0-53.0)$ \\
\hline ProGRP (pg/ml) & $8.5(0-28.5)$ & $17.4(0-46.5)$ \\
\hline
\end{tabular}

Tumor status at the operation was according to the TNM classification (UICC, 6th edition).

paraffin for sectioning at a thickness of $4 \mu \mathrm{m}$. Immunohistochemical reactions were carried out by the streptavidinbiotin-peroxidase methods. Sections were deparaffinized in xylene, and rehydrated through a graded series of ethanol. The specimens were boiled in target retrieval solution at $10 \mathrm{x}$ concentration (Dako Cytomation, Glostrup, Denmark; diluted at 1:10) in a pressure cooker for $8 \mathrm{~min}$. Endogenous peroxidase activity was blocked by incubation in $0.3 \%$ hydrogen peroxide methanol for $10 \mathrm{~min}$. The specimens were then washed with phosphate-buffered saline (PBS, $\mathrm{pH}$ 7.2), placed in a serum-free protein blocker (Dako corporation, Carpinteria, CA) for $5 \mathrm{~min}$, and incubated overnight at $4^{\circ} \mathrm{C}$ with the primary antibodies, anti-HOXA5 goat immunoglobulin polyclonal antibody and anti-HOXA10 goat immunoglobulin polyclonal antibody 

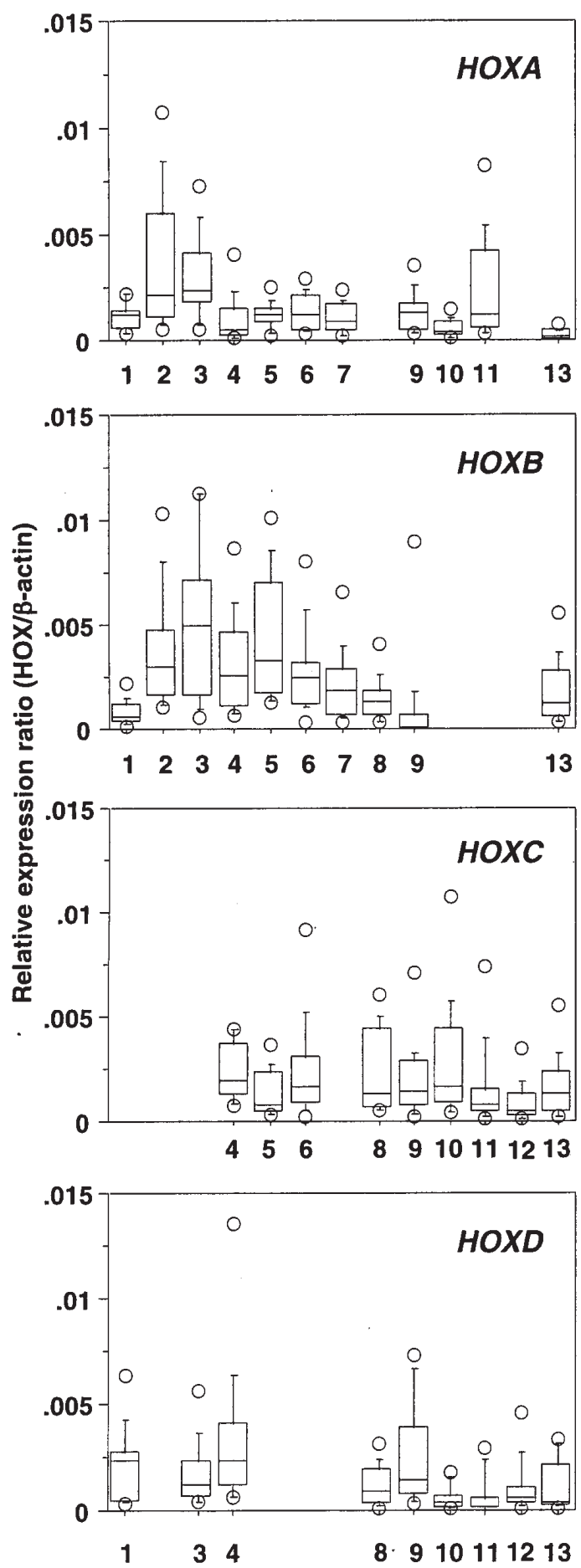

Figure 1. Expression profiling of $39 \mathrm{HOX}$ genes in non-cancerous tissues. The relative levels of HOX mRNA were determined by quantitative RTPCR in 15 of the non-cancerous tissues. The graphs of HOX cluster A, B, C and $\mathrm{D}$ are lined from top to bottom. HOX gene paralog 1-13 are lined from left to right of $\mathrm{x}$-axis in each graph. The distribution of the relative expression ratios (HOX/ß-actin) was summarized by using box-whisker graphs. The central box in each plot shows the interquartile (25th to 75th percentile) range. The line in the box shows the median. The whiskers (vertical bars) were drawn to the 90th and 10th percentiles. Extreme values greater than the 90 th percentile and less than the 10 th percentile were plotted individually.

(Santa Cruz Biotechnology, CA; diluted at 1:200). After washing in PBS, Biotinylated Link Universal (Dako Cytomation) was applied, and the specimens were incubated at room temperature for $20 \mathrm{~min}$. After washing in PBS, the specimens were incubated in streptavidin conjugated with peroxidase solution (Nichirei, Tokyo, Japan) at room temperature for $15 \mathrm{~min}$. After washing in PBS, immunohistochemical reactions were developed in freshly prepared 3,3'-diaminobenzidine tetrahydrochloride (Nichirei). The sections were counterstained with hematoxylin and coverslipped in a systemic mounting medium. As a negative control, we omitted the primary antibody.

Statistical analysis. The relationship between the expression of each HOX and each clinicopathological parameter was determined by the Mann-Whitney U test, the Kruskal-Wallis rank test or the Spearman rank correlation test. The statistical software package applied was StatView 5.0 for Macintosh (SAS Institute, Cary, NC). A p-value of $<0.01$ was considered statistically significant.

\section{Results}

Expressions of HOX genes in non-cancerous tissues. Fig. 1 shows the expression levels of 39 HOX genes in 15 noncancerous lung tissues which were obtained from the surgically resected tissues of lung cancer patients. The non-cancerous tissues showed relatively high expressions of HOXB2, B3, B4, B5 and B6 which were located at 3'-side of cluster B, and HOXA3 (median values of relative expression ratio were $>0.0025$ ) whereas they showed relatively low or no expression of Abd-B family of HOX genes (paralog 9-13) which were located at 5'-side of each cluster. Generally, there was a tendency that the expression levels varied relatively in a wide range within each sample.

Differences in HOX gene expression between lung cancer and non-cancerous tissues. We compared the expression levels of each HOX gene between lung cancer tissues and noncancerous tissues. Four of 39 HOX genes, HOXA1, A5, A10 and C6, showed significant differences in the expression levels between cancer and non-cancerous tissues $(p<0.01$, Mann-Whitney U test) (Fig. 2). The expression levels of all the four HOX genes in squamous cell carcinoma tissues were higher than those in non-cancerous tissues. In adenocarcinoma tissues, the expression levels of HOXA5 and A10 were high compared to non-cancerous tissues. We also found significant differences in the expression levels between squamous cell carcinoma and adenocarcinoma tissues: HOXA1, D9, D10 and D11 showed higher expressions in squamous cell carcinoma than in adenocarcinoma tissues (Fig. 3).

HOX gene expression and clinicopathologic parameters. We next examined whether there was a relationship between HOX gene expression patterns and clinicopathologic parameters. Although we did not find significant difference in any HOX gene expression between cancer tissues with metastasis to lymph node and those without, there was a tendency that the expression levels of HOXB7 in adenocarcinoma tissues with lymph node metastasis were low compared to those without $(\mathrm{p}=0.0295$, Mann-Whitney U test). There was no HOX gene which characterized the disease stage (IB, IIB and IIIA) or histological types of differentiation. We also examined the 

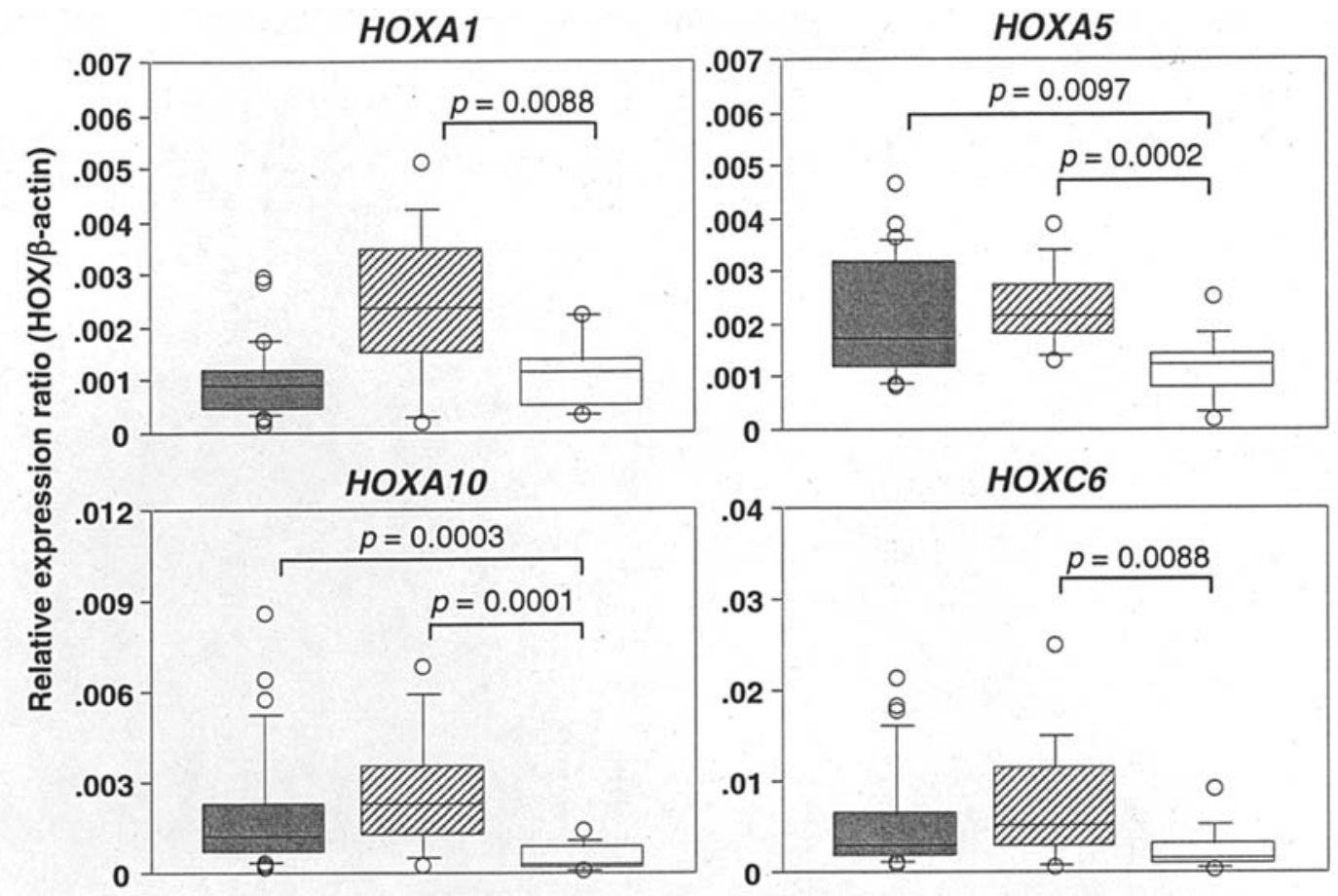

Figure 2. Different expression levels of HOX genes between non-SCLC and non-cancerous tissues. The distribution of the relative expression ratios (HOX/ $B$-actin) was summarized by using box-whisker plots in the same manner as in Figure 1. Shaded, hatched and open boxes represent adenocarcinoma, squamous cell carcinoma and non-cancerous tissues, respectively. p-values were determined by the Mann-Whitney U test.
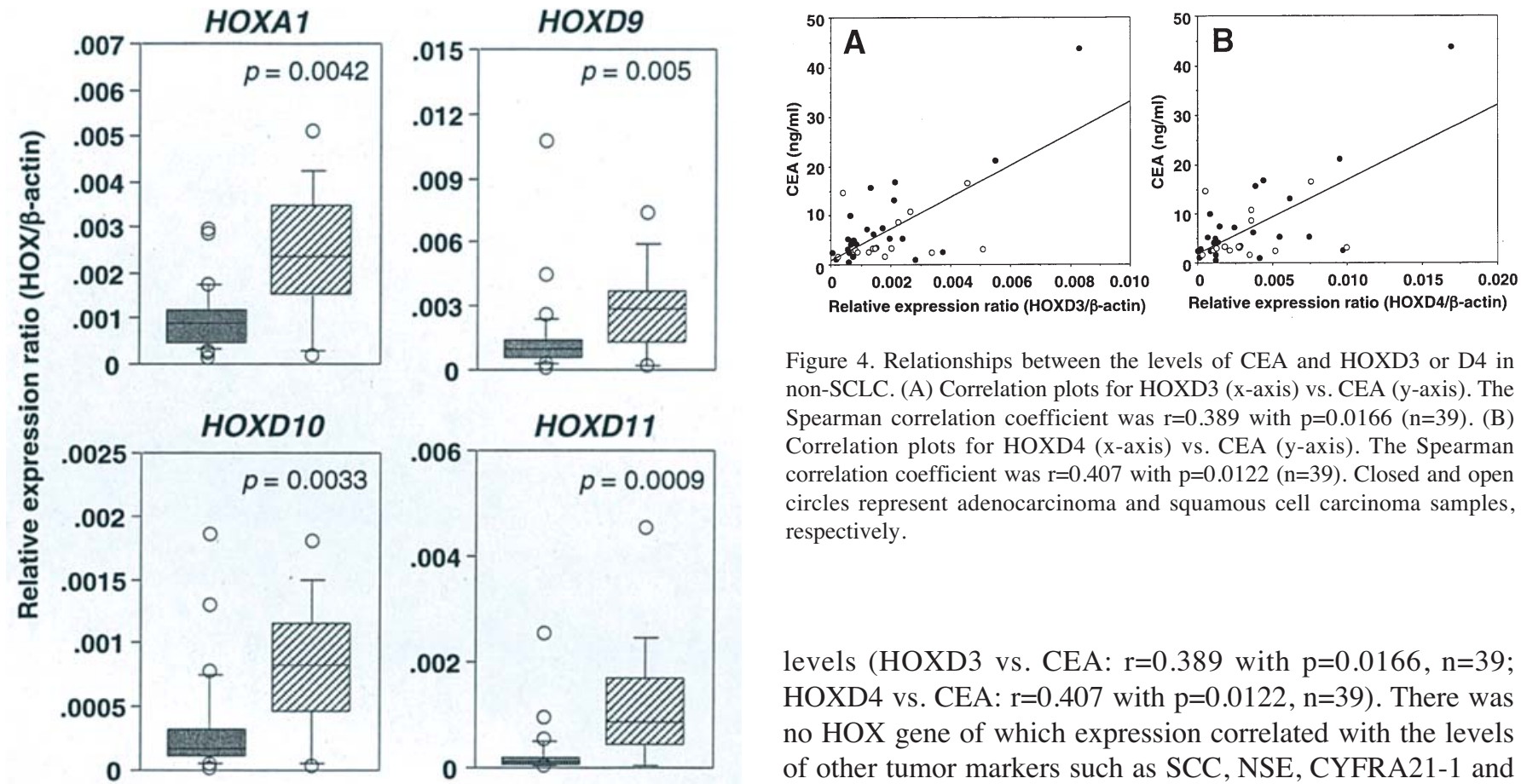

Figure 4. Relationships between the levels of CEA and HOXD3 or D4 in non-SCLC. (A) Correlation plots for HOXD3 (x-axis) vs. CEA (y-axis). The Spearman correlation coefficient was $r=0.389$ with $\mathrm{p}=0.0166(\mathrm{n}=39)$. (B) Correlation plots for HOXD4 (x-axis) vs. CEA (y-axis). The Spearman correlation coefficient was $r=0.407$ with $\mathrm{p}=0.0122(\mathrm{n}=39)$. Closed and open circles represent adenocarcinoma and squamous cell carcinoma samples, respectively.

Figure 3. Different expression levels of HOX genes between adenocarcinoma and squamous cell carcinoma tissues. The distribution of the relative expression ratios (HOX/B-actin) was summarized by using boxwhisker plots in the same manner as in Figure 1. Shaded and hatched boxes represent adenocarcinoma and squamous cell carcinoma, respectively. p-values were determined by the Mann-Whitney U test.

correlation between HOX gene expressions and tumor marker levels. As shown in Fig. 4, there was a slight correlation between CEA levels and HOXD3 or HOXD4 expression

levels (HOXD3 vs. CEA: $r=0.389$ with $\mathrm{p}=0.0166, \mathrm{n}=39$; HOXD4 vs. CEA: $r=0.407$ with $p=0.0122, n=39$ ). There was no HOX gene of which expression correlated with the levels of other tumor markers such as SCC, NSE, CYFRA21-1 and ProGRP or smoking.

Immunohistochemical examination for HOXA5 and A10 in non-SCLC specimens. We subjected non-SCLC tissues and their adjacent non-cancerous tissues of 8 specimens (4 adenocarcinoma and 4 squamous cell carcinoma) to immunohistochemical staining to detect the protein expression of HOXA5 and A10 of which expressions were higher at mRNA levels in non-SCLC than non-cancerous tissues. Fig. 5 shows representative examples of immunohistochemical staining for HOXA5 and A10. Immunoreactivity of HOXA5 and A10 

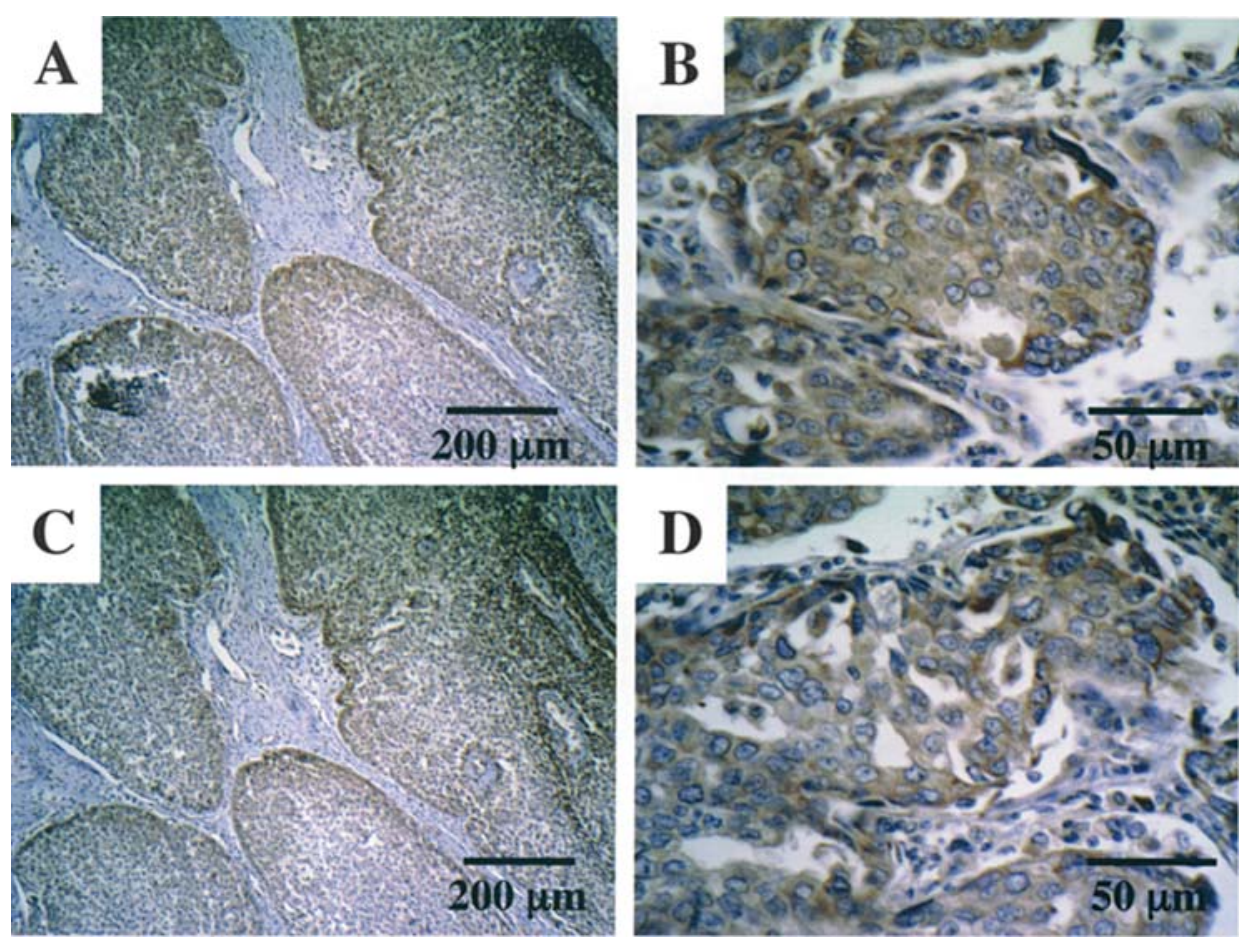

Figure 5. Immunohistochemical staining of HOXA5 and A10. Immunoreactivity of HOXA5 and A10 was observed in tumor cells but not in interstitial tissues (A, HOXA5; C, HOXA10, original magnification x40). Immunoreactivity of both HOXA5 and A10 was observed in the cytoplasm but not the nuclei of the tumor cells (B, HOXA5; D, HOXA10, original magnification x400).

was observed in cytoplasma of lung cancer cells and bronchial epithelial cells but neither in alveolar epithelial cells nor in lung cancer interstitial tissue. The immunoreactivity of both HOX proteins was detected in squamous metaplasia tissues involved in squamous cell carcinoma. Intensity of immunoreactivity of both $\mathrm{HOX}$ proteins was consistent with the expression levels of mRNA for the HOX genes in the 8 samples examined (data not shown).

\section{Discussion}

In this study, we tried to elucidate whether inappropriate expressions of HOX genes were involved in the development and progression of non-small cell lung cancer (non-SCLC). The comprehensive analysis of 39 HOX genes in 41 nonSCLC and 15 non-cancerous tissues revealed the expression patterns of HOX genes characteristic of adenocarcinoma or squamous cell carcinoma. Compared to non-cancerous tissue, adenocarcinoma tissue showed enhanced expressions of HOXA5 and A10, and squamous cell carcinoma tissue showed enhanced expressions of HOXA1 and C6 in addition to the two HOX genes. It is likely that the increased expressions of HOXA5 and/or A10 are positively associated with the development of non-SCLC. The HOXA5 gene product is a potent transactivator of the p53 promoter (16). The HOXA10 gene product activates p 21 transcription and results in cell cycle arrest and differentiation in myelomonocytic cells (17). These studies suggest anti-oncogenic function of both HOXA5 and A10 proteins, which is contradictory to our assumption of the roles of the two HOX genes in the development of nonSCLC. However, our immunohistological analysis suggested the possibility of this interpretation: Unexpectedly, both HOX proteins existed in the cytoplasm but not in the nuclei of cancer cells, indicating that they do not function as transcription factors regulating downstream target genes such as p53 and p21. HOX proteins are known to have other functions than DNA binding-transcription factors. For example, HOXA13 and D13 interact with Smad protein to modify Smad transcriptional activation (18). The homeodomain of HOX proteins binds to CREB binding protein (CBP) and blocks its acetyltransferase activity (19). These indicate that HOX proteins modulate the transcriptional activity through interaction with other proteins in a non-DNA binding manner. Therefore, we need to consider the possibility that HOXA5 and A10 proteins interfere with the function of anti-oncogenic molecules in lung cancer cells.

It is known that HOXA1 and C6, which were upregulated in squamous cell carcinoma, affect both cell growth and death. Forced expression of HOXA1 in human breast cancer cells results in increased cell growth activity (20). Loss of HOXC6 expression by transfection with siRNA induces apoptosis in human prostate cancer cell lines (21). Therefore, the increased expressions of HOXA1 and C6 may play a promoting role in the primary growth of lung squamous cell carcinoma tissues.

In adenocarcinoma and squamous cell carcinoma, 4 HOX genes presented different expressions. The expression levels of HOXA1, D9, D10 and D11 were significantly higher in squamous cell carcinoma than in adenocarcinoma. The expression levels of HOXD12 and D13 also tended to be high in squamous cell carcinoma $(\mathrm{p}=0.05$ and 0.01 , respectively, Mann-Whitney U test, data not shown). We examined the relationship between the expression of these HOX genes, and SCC and CYFRA21-1 which are tumor markers of lung 
squamous cell carcinoma. There was no relationship between them. As the expressions of HOXA1 and HOXD9-11 genes were also upregulated in oral squamous cell carcinoma (unpublished data), these HOX genes are likely involved in maintaining the squamous cell carcinoma-related phenotypes other than SCC and CYFRA21-1.

We found a positive correlation between CEA levels and HOXD3 or D4 expression levels although their correlation coefficients were not so high. There are many common phenomena between cancer and embryonic morphogenesis, one of which is the production of oncofetal proteins. This evidence provides the possibility to consider HOX genes as candidates which regulate the production of oncofetal proteins.

In conclusion, the results suggest that the disordered patterns of HOX gene expressions were involved not only in the development of non-SCLC but also in the histologically aberrant diversity such as adenocarcinoma and squamous cell carcinoma.

\section{Acknowledgements}

We wish to thank Ms. Masako Yanome for her help in preparing the report. This study was supported in part by Grantin-Aid for Scientific Research (B) (J.H.) from the Ministry of Education, Culture, Sports, Science and Technology of Japan.

\section{References}

1. Gehring WJ and Hiromi Y: Homeotic genes and the homeobox. Annu Rev Genet 20: 147-173, 1986.

2. McGinnis W and Krumlauf R: Homeobox genes and axial patterning. Cell 68: 283-302, 1992.

3. Levine M and Hoey T: Homeobox proteins as sequence-specific transcription factors. Cell 55: 537-540, 1988

4. Ruddle FH, Bartels JL, Bentley KL, Kappen C, Murtha MT and Pendleton JW: Evolution of Hox genes. Annu Rev Genet 28: 423-442, 1994.

5. Krumlauf R: Hox genes in vertebrate development. Cell 78: 191-201, 1994.

6. Cillo C, Barba P, Freschi G, Bucciarelli G, Magli MC and Boncinelli E: HOX gene expression in normal and neoplastic human kidney. Int J Cancer 51: 892-897, 1992.

7. De Vita G, Barba P, Odartchenko N, Givel JC, Freschi G, Bucciarelli G, Magli MC, Boncinelli E and Cillo C: Expression of homeobox-containing genes in primary and metastatic colorectal cancer. Eur J Cancer 29: 887-893, 1993.
8. Cantile M, Cindolo L, Napodano G, Altieri V and Cillo C: Hyperexpression of locus $\mathrm{C}$ genes in the HOX network is strongly associated in vivo with human bladder transitional cell carcinomas. Oncogene 22: 6462-6468, 2003.

9. Maeda K, Hamada J, Takahashi Y, Tada M, Yamamoto Y, Sugihara T and Moriuchi T: Altered expressions of HOX genes in human cutaneous malignant melanoma. Int J Cancer 114: 436-441, 2005.

10. Waltregny D, Alami Y, Clausse N, De Leval J and Castronovo V: Overexpression of the homeobox gene HOXC8 in human prostate cancer correlates with loss of tumor differentiation. Prostate 50: 162-169, 2002.

11. Tiberio C, Barba P, Magli MC, Arvelo F, Le Chevalier T, Poupon MF and Cillo C: HOX gene expression in human smallcell lung cancers xenografted into nude mice. Int J Cancer 58: 608-615, 1994.

12. Calvo R, West J, Franklin W, Erickson P, Bemis L, Li E, Helfrich B, Bunn P, Roche J, Brambilla E, Rosell R, Gemmill RM and Drabkin HA: Altered HOX and WNT7A expression in human lung cancer. Proc Natl Acad Sci USA 97: 12776-12781, 2000.

13. Lechner JF, Wang Y, Siddiq F, Fugaro JM, Wali A, Lonardo F, Willey JC, Harris CC and Pass HI: Human lung cancer cells and tissues partially recapitulate the homeobox gene expression profile of embryonic lung. Lung Cancer 37: 41-47, 2002.

14. Sobin LH and Witterkind C (eds): TNM classification of malignant tumours. 6th edition. Wiley-Liss, New York, pp97$103,2002$.

15. Takahashi Y, Hamada J, Murakawa K, Takada M, Tada M, Nogami I, Hayashi N, Nakamori S, Monden M, Miyamoto M, Katoh H and Moriuchi T: Expression profiles of 39 HOX genes in normal human adult organs and anaplastic thyroid cancer cell lines by quantitative real-time RT-PCR system. Exp Cell Res 293: 144-153, 2004

16. Raman V, Martensen SA, Reisman D, Evron E, Odenwald WF, Jaffee E, Marks J and Sukumar S: Compromised HOXA5 function can limit p53 expression in human breast tumours. Nature 405: 974-978, 2000.

17. Bromleigh VC and Freedman LP: p21 is a transcriptional target of HOXA10 in differentiating myelomonocytic cells. Genes Dev 14: 2581-2586, 2000.

18. Williams TM, Williams ME, Heaton JH, Gelehrter TD and Innis JW: Group 13 HOX proteins interact with the MH2 domain of R-Smads and modulate Smad transcriptional activation functions independent of HOX DNA-binding capability. Nucleic Acids Res 33: 4475-4484, 2005.

19. Shen WF, Krishnan K, Lawrence HJ and Largman C: The HOX homeodomain proteins block CBP histone acetyltransferase activity. Mol Cell Biol 21: 7509-7522, 2001.

20. Zhang X, Zhu T, Chen Y, Mertani HC, Lee KO and Lobie PE: Human growth hormone-regulated HOXA1 is a human mammary epithelial oncogene. J Biol Chem 278: 7580-7590, 2003.

21. Ramachandran S, Liu P, Young AN, Yin-Goen Q, Lim SD, Laycock N, Amin MB, Carney JK, Marshall FF, Petros JA and Moreno CS: Loss of HOXC6 expression induces apoptosis in prostate cancer cells. Oncogene 24: 188-198, 2005. 